\title{
GENERALIZED CLOSE-TO-CONVEXITY RELATED WITH BOUNDED BOUNDARY ROTATION
}

\section{KHALIDA INAYAT NOOR ${ }^{1}$, MUHAMMAD ASLAM NOOR ${ }^{1, *}$ AND MUHAMMAD UZAIR AWAN ${ }^{2}$}

\author{
${ }^{1}$ COMSATS University Islamabad, Islamabad, Pakistan \\ ${ }^{2}$ Government College University Faisalabad, Pakistan
}

${ }^{*}$ Corresponding author: noormaslam@gmail.com

\begin{abstract}
The class $P_{\alpha, m}[A, B]$ consists of functions $p$, analytic in the open unit disc $E$ with $p(0)=1$ and satisfy

$$
p(z)=\left(\frac{m}{4}+\frac{1}{2}\right) p_{1}(z)-\left(\frac{m}{4}-\frac{1}{2}\right) p_{2}(z), \quad m \geq 2,
$$

and $p_{1}, p_{2}$ are subordinate to strongly Janowski function $\left(\frac{1+A z}{1+B z}\right)^{\alpha}, \alpha \in(0,1]$ and $-1 \leq B<A \leq 1$. The class $P_{\alpha, m}[A, B]$ is used to define $V_{\alpha, m}[A, B]$ and $T_{\alpha, m}\left[A, B ; 0 ; B_{1}\right], B_{1} \in[-1,0)$. These classes generalize the concept of bounded boundary rotation and strongly close-to-convexity, respectively. In this paper, we study coefficient bounds, radius problem and several other interesting properties of these functions. Special cases and consequences of main results are also deduced.
\end{abstract}

\section{INTRODUCTION}

Let $A$ denote the class of analytic functions defined in the open unit disc $E=\{z:|z|<1\}$ and be given by

$$
f(z)=z+\sum_{n=2}^{\infty} a_{n} z^{n}, \quad z \in E
$$

Received August $6^{\text {th }}, 2021$; accepted September 23 ${ }^{\text {rd }}, 2021$; published October $28^{\text {th }}, 2021$.

2010 Mathematics Subject Classification. 30C45.

Key words and phrases. Janowski function; Subordination bounded boundary rotation; univalent; starlike; close-to-convex; integral operator; coefficient problem.

(C)2021 Authors retain the copyrights of their papers, and all open access articles are distributed under the terms of the Creative Commons Attribution License. 
Let $S \subset A$ be the class of univalent functions in $E$ and let $C, S^{\star}$ and $K$ be the subclasses of $S$ consisting of convex, starlike and close-to-convex functions, respectively. For details, see [3].

For $f, g \in A$, we say $f$ is subordinate to $g$ in $E$, written as $f(z) \prec g(z)$, if there exists a Schwartz function $w(z)$ such that

$$
f(z)=g(w(z)), \quad g(z)=z+\sum_{n=2}^{\infty} b_{n} z^{n} .
$$

Furthermore, if the function $g$ is univalent in $E$, then we have the following equivalence

$$
f(z) \prec g(z) \Leftrightarrow f(0)=g(0) \text { and } f(E) \subset g(E) .
$$

Convolution of $f$ and $g$ is defined as

$$
(g * f)(z)=z+\sum_{n=2}^{\infty} a_{n} b_{n} z^{n} .
$$

The class $P_{\alpha}[A, B]$ of strongly Janowski functions is defined as follows.

Definition 1.1. Let $p$ be analytic in $E$ with $p(0)=1$. Then $p \in P_{\alpha}[A, B]$, if $p(z) \prec\left(\frac{1+A z}{1+B z}\right)^{\alpha}$, where $\alpha \in(0,1]$ and $-1 \leq B<A \leq 1$.

We denote $P_{\alpha}\left[0, B_{1}\right]$ as $P_{\alpha}\left[B_{1}\right],-1 \leq B_{1}<0$.

The class $P_{\alpha}[A, B]$ is generalized as:

Definition 1.2. An analytic function $p: p(z)=1+\sum_{n=1}^{\infty} c_{n} z^{n}$ is in the class $P_{\alpha, m}[A, B]$, if and only if, there exist $p_{1}, p_{2} \in P_{\alpha}[A, B]$ such that

$$
p(z)=\left(\frac{m}{4}+\frac{1}{2}\right) p_{1}(z)-\left(\frac{m}{4}-\frac{1}{2}\right) p_{2}(z), \quad m \geq 2 .
$$

It is obvious $P_{\alpha, 2}[A, B]=P_{\alpha}[A, B]$. For the class $P_{1}[A, B]=P[A, B]$, we refer to $[6]$. About the class $P_{\alpha}[A, B]$, we observe the following.

(i) $p(z) \prec\left(\frac{1+A z}{1+B z}\right)^{\alpha}$ implies $p \in P_{\alpha}[A, B]$ and it can easily be shown that $\phi_{\alpha}(A, B ; z)=\left(\frac{1+A z}{1+B z}\right)^{\alpha}$ is convex univalent in $E$. In fact simple calculation yield that

$$
\operatorname{Re}_{\alpha}^{\prime}(A, B ; z) \geq \alpha|A-B| \frac{(1-|A|)^{\alpha-1}}{(1-|B|)^{\alpha+1}}>0, \quad z \in E .
$$

This shows $\phi_{\alpha}(A, B ; z)$ is univalent in $E$.

Also

$$
\operatorname{Re}\left\{\frac{\left(z \phi_{\alpha}^{\prime}(A, B ; z)\right)^{\prime}}{\phi_{\alpha}^{\prime}(A, B ; z)}\right\} \geq \frac{T(r)}{(1+A r)(1+B r)}
$$

where

$$
T(r)=1-\alpha(A-B) r-A B r^{2}
$$


is decreasing on $(0,1)$ and $T(0)=1$.

This implies $\operatorname{Re}\left[\frac{\left(z \phi_{\alpha}^{\prime}(A, B ; z)\right)^{\prime}}{\phi_{\alpha}^{\prime}(A, B ; z)}\right] \geq 0$ in $E$.

(ii) For $A=1, B=-1, p \in \phi_{\alpha}(1,-1 ; z)$ implies

$$
|\arg p(z)| \leq \frac{\alpha \pi}{2}, \quad z \in E
$$

Definition 1.3. Let $f, g \in A, \frac{(g * f)^{\prime}(z)}{z} \neq 0, z \in E$. Then $f \in V_{\alpha, m}[A, B ; g]$, if and only if,

$$
\frac{\left(z(g * f)^{\prime}\right)^{\prime}}{(g * f)^{\prime}} \in P_{\alpha, m}[A, B], \quad z \in E
$$

with $F=z f^{\prime}, F \in R_{\alpha, m}[A, B ; g]$, if and only if, $f \in V_{\alpha, m}[A, B ; g]$ in $E$.

Special Cases.

(i) $V_{1, m}\left[A, B ; \frac{z}{1-z}\right]=V_{m}[A, B] \subset V_{m}[1,-1]=V_{m}$, where $V_{m}$ is the well known class of functions of bounded boundary rotation. See, for example, [2,10,12].

(ii) $R_{1, m}\left[A, B ; \frac{z}{1-z}\right]=R_{m}[A, B] \subset R_{m}$ and $R_{m}$ is the class of functions with bounded radius rotation, see $[9]$.

(iii) $V_{\alpha, m}\left[A, B ; \frac{z}{(1-z)^{2}}\right]=R_{\alpha, m}\left[A, B ; \frac{z}{1-z}\right]=R_{\alpha, m}[A, B]$.

Definition 1.4. Let $f, g \in A$ with $(g * f)(z) \neq 0$. Then $f \in T_{\alpha, m}\left[A, B ; 0 ; B_{1} ; g\right]$, if there exists $\psi \in$ $V_{\alpha, m}[A, B ; g]$ such that, for $B_{1} \in[-1,0)$,

$$
\frac{(g * f)^{\prime}}{(g * \psi)^{\prime}} \in P_{\alpha}\left[B_{1}\right], \quad z \in E
$$

We note that $T_{1}\left[A, B ; 0 ;-1 ; \frac{z}{1-z}\right]=T_{m}[A, B]$. For certain special cases, see $[8,11,12]$.

2. THE CLASS $V_{\alpha, m}[A, B ; g]$

Theorem 2.1. Let $f \in V_{\alpha, m}[A, B ; g]$ and let $g(z)=z+\sum_{n=2}^{\infty} b_{n} z^{n}$. Then, with $f$ given by $(1.1), A_{n}=a_{n} b_{n}$,

$$
A_{n}=O(1) n^{\sigma}, \quad \sigma=\left\{\left(\frac{m}{2}+1\right)(1-\rho)-(\rho+2)\right\}
$$

where $\rho=\left(\frac{1-A}{1-B}\right)^{\alpha}, \quad m \geq \frac{2(1+\rho)}{1-\rho}$ and $O(1)$ denotes a constant.

Proof. Let $F=f * g$. Then $F \in V_{\alpha, m}[A, B]$. Since $p \in P_{\alpha}[A, B]$ implies $\operatorname{Re} p(z)>\rho, \rho=\left(\frac{1-A}{1-B}\right)^{\alpha}$, it follows that $V_{\alpha, m}[A, B] \subset V_{m}(\rho)$.

Now, $F \in V_{m}(\rho)$, we can write

$$
F_{1}^{\prime}(z)=\left(F_{1}^{\prime}(z)\right)^{1-\rho}, \quad F_{1} \in V_{m}
$$


see [13].

Using a result due to Brannan [2], we can write

$$
z F_{1}^{\prime}(z)=\frac{\left(s_{1}(z)\right)^{\left(\frac{m}{4}+\frac{1}{2}\right)(1-\rho)}}{\left(s_{2}(z)\right)^{\left(\frac{m}{4}-\frac{1}{2}\right)(1-\rho)}}, \quad s_{1}, s_{2} \in S^{\star} .
$$

Therefore, from (2.1), (2.2) and Cauchy Theorem with $z=r e^{\iota \theta}$, we have

$$
\begin{aligned}
n^{2}\left|A_{n}\right| & \leq \frac{1}{2 \pi r^{n}} \int_{0}^{2 \pi}\left|F_{1}^{\prime}(z) h(z)\right|^{1-\rho} d \theta, \quad h \in P_{\alpha, m}[A, B] \subset P_{m}(\rho) \\
& =\frac{1}{2 \pi r^{n+1}} \int_{0}^{2 \pi} \frac{\left|s_{1}(z)\right|^{\left(\frac{m}{4}+\frac{1}{2}\right)(1-\rho)}}{\left|s_{2}(z)\right|^{\left(\frac{m}{4}-\frac{1}{2}\right)(1-\rho)}} \cdot|h(z)|^{1-\rho} d \theta
\end{aligned}
$$

Applying distortion result for $s_{2} \in S^{\star}$ and Holder's inequality in (2.3), we get

$$
\begin{aligned}
n^{2}\left|A_{n}\right| & \leq \frac{1}{r^{n+1}}\left(\frac{4}{r}\right)^{\left(\frac{m}{4}-\frac{1}{2}\right)(1-\rho)}\left(\frac{1}{2 \pi} \int_{0}^{2 \pi}\left|s_{1}(z)\right|^{\left\{\left(\frac{m}{4}+\frac{1}{2}\right)(1-\rho)\right\} \frac{2}{1+\rho}} d \theta\right)^{\frac{1+\rho}{2}} \\
& \cdot\left(\frac{1}{2 \pi} \int_{0}^{2 \pi}|h(z)|^{2} d \theta\right)^{\frac{1-\rho}{2}}
\end{aligned}
$$

Now, for $h(z)=1+\sum_{n=1}^{\infty} c_{n} z^{n}$, we use Parsval identity to have

$$
\begin{aligned}
\frac{1}{2 \pi} \int_{0}^{2 \pi}|h(z)|^{2} d \theta & =\sum_{n=0}^{\infty}\left|c_{n}\right|^{2} r^{2 n} \\
& \leq 1+m^{2}(1-\rho)^{2} \sum_{n=1}^{\infty} r^{2 n} \\
& =\frac{1+\left[m^{2}(1-\rho)^{2}-1\right] r^{2}}{1-r^{2}}
\end{aligned}
$$

where we have used coefficient bounds $\left|c_{n}\right| \leq m(1-\rho)$, for $h \in P_{m}(\rho)$.

From (2.5) together with subordination for starlike functions, and a result due to Hayman [5] for $m \geq \frac{2(1+\rho)}{1-\rho}$, we have

$$
n^{2}\left|A_{n}\right| \leq c_{1}(m, \rho)\left(\frac{1}{1-r}\right)^{\left\{\left(\frac{m}{2}+1\right)(1-\rho)\right\}-\rho},
$$

where $c_{1}(m, \rho)$ denotes a constant.

Taking $r=1-\frac{1}{n}$ in (2.6), we obtain the required result.

\section{Special Cases.}

(i) Let $g(z)=\frac{z}{1-z}$, then $A_{n}=a_{n}$. Take $A=0$, and in this case $f \in V_{m}$. This leads us to a known coefficient result that $a_{n}=O(1) n^{\left(\frac{m}{2}-1\right)}$.

(ii) Let $f \in V_{1, m}\left[0,-1, \frac{z}{(1-z)^{2}}\right]=R_{m}\left(\frac{1}{2}\right)$. Then $a_{n}=O(1) n^{\frac{m}{4}-2}, m \geq 6$. 
Theorem 2.2. Let $f \in V_{\alpha, m}[A, B ; g]$. Then, for $F=f * g, z=r e^{\iota \theta}$,

$0 \leq \theta_{1}<\theta_{2} \leq 2 \pi$, we have

$$
\int_{\theta_{1}}^{\theta_{2}} \operatorname{Re}\left\{\frac{\left(z F^{\prime}(z)\right)^{\prime}}{F^{\prime}(z)}\right\} d \theta>-\left(\frac{m}{2}-1\right)(1-\rho) \pi, \quad \rho=\left(\frac{1-A}{1-B}\right)^{\alpha} .
$$

Proof. Proof is straight forward, since $V_{\alpha, m}[A, B] \subset V_{m}(\rho)$ and $F \in V_{m}(\rho)$ implies there exist $F_{1} \in V_{m}$ with $F^{\prime}(z)=\left(F_{1}^{\prime}(z)\right)^{(1-\rho)}$. Now, using essentially the same method given in [2], the required result follows.

Remark 2.1. Let $\beta\left(\frac{m}{2}-1\right)(1-\rho)$. Then, from a result of Goodman [4] and from (2.7), it follows that $F=f * g \in V_{\alpha, m}[A, B]$ is univalent for $\beta=\left(\frac{m}{2}-1\right)(1-\rho) \leq 1$. That is $F \in S$ for $m \leq \frac{2(2-\rho)}{1-\rho}$. As a special case, with $g(z)=\frac{z}{1-z}, A=0, B=-1$ and $\alpha=1$, we have $F=f, \rho=\frac{1}{2}$. Then $f \in V_{1, m}[0,-1]$ implies

$$
\int_{\theta_{1}}^{\theta_{2}} \operatorname{Re}\left\{\frac{\left(z F^{\prime}(z)\right)^{\prime}}{F^{\prime}(z)}\right\} d \theta>-\left(\frac{m}{4}-\frac{1}{2}\right) \pi
$$

For this, we can conclude that

$$
V_{1, m}[0,-1] \subset S \quad \text { for } \quad 2 \leq m \leq 6
$$

Also, with $g(z)=\frac{z}{1-z}, A=1, B=-1$, we have a well known result that $f \in V_{m}$ is univalent for $2 \leq m \leq 4$.

Theorem 2.3. Let $f \in V_{\alpha, m}[A, B ; g], m \leq \frac{2(2-\rho)}{1-\rho}$ and $\rho=\left(\frac{1-A}{1-B}\right)^{\alpha}$. Then $F(E)$ with $F=f * g$, contains the disc d:

$$
d=\left\{w:|w|<\frac{4}{8+\alpha m|A-B|}\right\}
$$

Proof. From Theorem 2.2, $F$ is univalent in $E$. Let $w_{0}\left(w_{0} \neq 0\right)$ be any complex number such that $F(z) \neq w_{0}$ for $z \in E$. Then the function

$$
F_{1}(z)=\frac{w_{0} F(z)}{w_{0}-F(z)}=z+\left(A_{2}+\frac{1}{w_{0}}\right) z^{2}+\ldots
$$

is analytic and univalent in $E$. Using the well known Bieberbach Theorem for the best bound for second coefficient of univalent functions, see [3], we have

$$
\frac{1}{\left|w_{0}\right|}-\left|A_{2}\right| \leq\left|A_{2}+\frac{1}{w_{0}}\right| \leq 2 .
$$

This gives us

$$
\begin{aligned}
\frac{1}{\left|w_{0}\right|} & \leq 2+\left|A_{2}\right| \\
& \leq 2+\frac{\alpha_{m}|A-B|}{4}=\frac{8+\alpha_{m}|A-B|}{4} .
\end{aligned}
$$

This completes the proof. 


\section{Special Cases.}

(i) Let $A=1, B=-1, \alpha=1 ;(\rho=0)$ and so $F(E)$ contains the disc $|w|<\frac{2}{4+m}, m \leq 4$.

(ii) With $A=0, B=-1, \alpha=\frac{1}{2}$, we have $\rho=\frac{1}{4}$, and $F(E)$ contains the disc $|w|<\frac{8}{16+m}, m \leq \frac{14}{3}$.

The following properties of the class $V_{\alpha, m}[A, B ; g]$ can easily be proved with simple computations and well known results and therefore we omit the proof.

Theorem 2.4. $\quad$ (i) The class $V_{\alpha, m}[A, B ; g]$ is preserved under the integral operator $L: A \rightarrow A$ defined as

$$
L(z)=\int_{0}^{z}\left(L_{1}^{\prime}(\xi)\right)^{\beta}\left(L_{2}^{\prime}(\xi)\right)^{\gamma} d \xi
$$

where $L_{i} \in V_{\alpha, m}[A, B ; g], i=1,2$ and $\beta, \gamma$ are positively real with $\beta+\gamma=1$.

(ii) Let $f \in V_{\alpha, m}\left[A, B ; \frac{z}{1-z}\right]$. Then, with $\rho=\left(\frac{1-A}{1-B}\right)^{\alpha}, z \in E$ and $z=r e^{\iota \theta}$, we have

$$
\frac{(1-B r)^{(1-\rho)\left(\frac{m}{4}+\frac{1}{2}\right)}}{(1+B r)^{(1-\rho)\left(\frac{m}{4}-\frac{1}{2}\right)}} \leq\left|f^{\prime}(z)\right| \leq \frac{(1+B r)^{(1-\rho)\left(\frac{m}{4}+\frac{1}{2}\right)}}{(1-B r)^{(1-\rho)\left(\frac{m}{4}-\frac{1}{2}\right)}} .
$$

For $\alpha=1, f \in V_{m}[A, B]$ and $A=1, B=-1$, the result reduces to $f \in V_{m}$ studied in [2].

(iii) Let $f \in V_{\alpha, 2}\left[A, B ; \frac{z}{1-z}\right]$ and define $F \in A$ as

$$
F(z)=\frac{\beta+1}{z^{\beta}} \int_{0}^{z} t^{\beta-1} f(t) d t, \beta>0
$$

Then $F$ is convex of order $\gamma(\rho), \rho=\left(\frac{1-A}{1-B}\right)^{\alpha}$, where

$$
\gamma=\gamma(\rho)=\left\{\frac{(\beta+1)}{{ }_{2} F_{1}\left(2(1-\rho), 1 ;(\beta+2) ; \frac{1}{2}\right)}-\beta\right\},
$$

${ }_{2} F_{1}$ represents Gauss hypergeometric function.

(iv) The set of all points $\log f^{\prime}(z)$ for a fixed $z \in E$ and $f$ ranging over the class $V_{\alpha, m}[A, B ; g]$ is convex.

(v) Let $f \in V_{\alpha, m}\left[A, B ; \frac{z}{1-z}\right], B \neq 0$. Then $f$ is close-to-convex for $|z|<r_{1}$, where

$$
r_{1}=\left\{\sin \left(\frac{\pi}{B(\gamma-2)}\right), \quad B \neq 0, \quad m>\frac{2}{\gamma}, \quad \gamma=1-\left(\frac{1-A}{1-B}\right)^{\alpha}\right.
$$

(vi) Let $f \in V_{\alpha, m}[A, B ; g]$, and let $F=f * g$. Then $F$ is convex of order $\rho=\left(\frac{1-A}{1-B}\right)^{\alpha}$ for $|z|<r_{m}$, where

$$
r(m)=\frac{m-\sqrt{m^{2}-4}}{2}, \quad m \geq 2
$$

Theorem 2.5. Let $f_{1}, f_{2} \in V_{\alpha, m}[A, B ; g], \beta, \delta, c$ and $\nu$ be positively real, $c \geq \beta \geq 1,(\nu+\delta)=\beta$. Let $F=F_{1} * g, G_{i}=f_{i} * g, i=1,2$ and define

$$
[F(z)]^{\beta}=c z^{(\beta-c)} \int_{0}^{z} t^{c-1}\left(G_{1}^{\prime}(t)\right)^{\delta}\left(G_{2}^{\prime}(t)\right)^{\nu} d t .
$$


Then, for $z=r e^{\iota \theta}, 0 \leq \theta_{1}<\theta_{2} \leq 2 \pi, \frac{z F^{\prime}}{F}=p$, we have

$$
\int_{\theta_{1}}^{\theta_{2}} \operatorname{Re}\left\{p(z)+\frac{\frac{1}{\beta} z p^{\prime}(z)}{p(z)+\frac{1}{\beta}(c-\beta)}\right\} d \theta>-(1-\rho)\left(\frac{m}{2}-1\right) \pi, \quad \rho=\left(\frac{1-A}{1-B}\right)^{\alpha} .
$$

Proof. First we show that there exists a function $F \in A$ satisfying (2.8). We assume $F_{1} * g \neq 0, f_{i} * g \neq 0$, $z \in E$. Let

$$
Q(z)=\left(G_{1}^{\prime}(z)\right)^{\delta}\left(G_{2}^{\prime}(z)\right)^{\nu}=1+d_{1} z+d_{2} z^{2}+\ldots
$$

and choose the branches which equal 1 , when $z=0$.

For $K(z)=z^{c-1}\left(G_{1}^{\prime}(z)\right)^{\delta}\left(G_{2}^{\prime}(z)\right)^{\nu}=z^{c-1} Q(z)$, we have

$$
N(z)=\frac{c}{z^{c}} \int_{0}^{z} K(t) d t=1+\frac{c}{c+1} d_{1} z+\ldots
$$

Hence $N$ is well defined and analytic.

Now let

$$
F(z)=\left[z^{\beta} N(z)\right]^{\frac{1}{\beta}}=z[N(z)]^{\frac{1}{\beta}},
$$

where we choose the branch of $[N(z)]^{\frac{1}{\beta}}$ which equal 1 when $z=0$. Thus $F \in A$ and satisfies (2.8). We write

$$
\frac{z F^{\prime}(z)}{F(z)}=p(z), \quad F=F_{1} * g
$$

From (2.8) and (2.9) with some calculations

$$
\beta p(z)+\frac{\beta z p^{\prime}(z)}{(c-\beta)+\beta p(z)}=\delta\left[\frac{\left(z G_{1}^{\prime}(z)\right)^{\prime}}{G_{1}^{\prime}(z)}\right]+\nu\left[\frac{\left(z G_{1}^{\prime}(z)\right)^{\prime}}{G_{2}^{\prime}(z)}\right] .
$$

That is

$$
p(z)+\frac{\frac{1}{\beta} z p^{\prime}(z)}{p(z)+\frac{1}{\beta}(c-\beta)}=\frac{]}{\delta} \beta\left[\frac{\left(z G_{1}^{\prime}(z)\right)^{\prime}}{G_{1}^{\prime}(z)}\right]+\frac{\nu}{\beta}\left[\frac{\left(z G_{1}^{\prime}(z)\right)^{\prime}}{G_{2}^{\prime}(z)}\right] .
$$

We now apply Theorem 2.2 and obtain the required result.

For $m \leq \frac{2(2-\rho)}{1-\rho}$ and applying a result proved in [14], it can easily be deduced that

$$
\int_{\theta_{1}}^{\theta_{2}} \operatorname{Re}\{p(z)\} d \theta>-\pi, \quad p(z)=\frac{z\left(F_{1} * g\right)^{\prime}}{F_{1} * g} .
$$

Taking $g(z)=\frac{z}{(1-z)^{2}}$, it follows that $F_{1} \in S$ in $E$, see [4].

3. The Class $T_{\alpha, m}\left[A, B ; 0 ; B_{1} ; g\right]$

Theorem 3.1. Let $f \in T_{\alpha, m}\left[A, B ; 0 ; B_{1} ; \frac{z}{1-z}\right]=T_{\alpha, m}\left[A, B ; 0 ; B_{1}\right]$. Then, for $z=r e^{\iota \theta}, 0 \leq \theta_{1}<\theta_{2} \leq 2 \pi$, $\rho_{1}=\left(\frac{1}{2}\right)^{\alpha}, \rho=\left(\frac{1-A}{1-B}\right)^{\alpha}$,

$$
\int_{\theta_{1}}^{\theta_{2}} \operatorname{Re}\left\{\frac{\left(z f^{\prime}(z)\right)^{\prime}}{f^{\prime}(z)}\right\} d \theta>-\beta \pi, \quad \beta=\left[\left(1-\rho_{1}\right)+\left(\frac{m}{2}-1\right)(1-\rho)\right] .
$$


Proof. For $f \in T_{\alpha, m}\left[A, B ; 0 ; B_{1}\right]$, we can write

$$
\frac{f^{\prime}(z)}{\psi^{\prime}(z)}=h(z), \quad \psi \in V_{\alpha, m}[A, B], \quad h \in P_{\alpha}\left[0, B_{1}\right] .
$$

To prove this result, we shall essentially follow the method due to Kaplan [4].

For $\psi \in V_{\alpha, m}[A, B]$, it implies that $\psi \in V_{m}(\rho)$, where $\rho=\left(\frac{1-A}{1-B}\right)^{\alpha}$.

Also $h \in P_{\alpha}\left[0, B_{1}\right], B_{1} \in[-1,0)$ is equivalent to $h \prec\left(\frac{1}{1+B_{1} z}\right)^{\alpha}$. That is, $h \in P\left(\alpha_{1}\right) \subset P, \alpha_{1}=\left(\frac{1}{2}\right)^{\alpha}$.

Now, with $z=r e^{\iota \theta}$, write $p(z)=\arg f^{\prime}(z)$ and $q(z)=\arg \psi^{\prime}(z)$. Then

$$
|p(z)-q(z)|<\left(1-\left(\frac{1}{2}\right)^{\alpha}\right) \frac{\pi}{2}
$$

Let $P(r, \theta)=p\left(r e^{\iota \theta}\right)+\theta, \quad Q(r, \theta)=q\left(r e^{\iota \theta}\right)+\theta$ be defined for $0 \leq r<1$ and for all $\theta$. This gives us

$$
|P(r, \theta)-Q(r, \theta)|<\left(1-\left(\frac{1}{2}\right)^{\alpha}\right) \frac{\pi}{2} .
$$

From Theorem 2.2, for $\psi \in V_{\alpha, m}[A, B] \subset V_{m}(\rho)$, we have

$$
\int_{\theta_{1}}^{\theta_{2}} \operatorname{Re}\left\{\frac{\left(z \psi^{\prime}(z)\right)^{\prime}}{\psi^{\prime}(z)}\right\} d \theta>-\left(\frac{m}{2}-1\right)\left(1-\left(\frac{1-A}{1-B}\right)^{\alpha}\right) \pi, \quad\left(z=r e^{\iota \theta}\right) .
$$

Thus

$$
\left|Q\left(r, \theta_{1}\right)-Q\left(r, \theta_{2}\right)\right|<\left(1-\left(\frac{1-A}{1-B}\right)^{\alpha}\right)\left(\frac{m}{2}-1\right) \pi .
$$

From (3.2) and (3.3), it follows that

$$
\begin{aligned}
& \left|P\left(r, \theta_{1}\right)-P\left(r, \theta_{2}\right)\right| \\
& =\left|\left\{P\left(r, \theta_{1}\right)-Q\left(r, \theta_{1}\right)\right\}-\left\{P\left(r, \theta_{2}\right)-Q\left(r, \theta_{2}\right)\right\}+\left\{Q\left(r, \theta_{1}\right)-Q\left(r, \theta_{2}\right)\right\}\right| \\
& <\left(1-\left(\frac{1}{2}\right)^{\alpha}\right) \frac{\pi}{2}+\left(1-\left(\frac{1}{2}\right)^{\alpha}\right) \frac{\pi}{2}+\left(1-\left(\frac{1-A}{1-B}\right)^{\alpha}\right)\left(\frac{m}{2}-1\right) \pi \\
& =\left[\left(1-\left(\frac{1}{2}\right)^{\alpha}\right)+\left(1-\left(\frac{1-A}{1-B}\right)^{\alpha}\right)\left(\frac{m}{2}-1\right)\right] \pi \\
& =\left[\left(1-\rho_{1}\right)+\left(\frac{m}{2}-1\right)(1-\rho)\right] \pi=\beta \pi,
\end{aligned}
$$

and this proves our result.

\section{Special Cases.}

(i) Let $\alpha=1, A=1$ and $B=-1$. Then $\beta=\frac{m-1}{2}=1$ for $m=3$. This implies $f \in T_{1, m}[1,-1 ; 0 ;-1]$ is univalent for $2 \leq m \leq 3$.

(ii) For $A=0, B=-1, \alpha=1$ we have $\beta=\frac{m}{4}$ and, in this case, $f$ is univalent for $2 \leq m \leq 4$. 
Remark 3.1. For $F \in A$, Goodman [4] introduced a class $K(\beta)$ as

$$
\int_{\theta_{1}}^{\theta_{2}} \operatorname{Re}\left\{\frac{\left(z F^{\prime}(z)\right)^{\prime}}{F^{\prime}(z)}\right\} d \theta>-\beta \pi, \quad z=r e^{\iota \theta}, \quad 0 \leq \theta_{1}<\theta_{2} \leq 2 \pi,
$$

and $\beta \geq 0$. When $0 \leq \beta \leq 1, K(\beta)$ consists of univalent functions

(close-to-convex), whilest for $\beta>1, F$ need not even be finitely-valent, see [4].

We note that, for $\rho_{1}=\left(\frac{1}{2}\right)^{\alpha}, \rho=\left(\frac{1-A}{1-B}\right)^{\alpha}$.

$$
T_{\alpha, m}\left[A, B, 0, B_{1}\right] \subset K\left(\frac{m}{2}(1-\rho)+\left(\rho-\rho_{1}\right)\right) .
$$

This implies $F \in T_{\alpha, m}[A, B ; 0 ;-1]$ is univalent for $m \leq 2\left[1+\frac{\rho_{1}}{1-\rho}\right]$.

Theorem 3.2. For $g(z)=\frac{z}{1-z}$, let $f \in T_{\alpha, 2}\left[A, B, 0, B_{1}\right]$ and for $\gamma, \beta>0$, let

$F_{1}$ be defined by

$$
F_{1}(z)=\left[(1+\beta) z^{-\beta} \int_{0}^{z} t^{\beta-1} f^{\gamma}(t) d t\right]^{\frac{1}{\gamma}} .
$$

Then $F_{1} \in T_{1,2}\left[A, B ; 0 ; B_{1}\right]$ in $E$.

Proof. We can write (3.4) as

$$
F_{1}(z)=\left[\left(\frac{f(z)}{z}\right)^{\gamma} *\left(\frac{\phi_{\gamma, \beta}(z)}{z}\right)\right]^{\frac{1}{\gamma}}
$$

where

$$
\phi_{\gamma, \beta}(z)=\sum_{n=1}^{\infty}\left(\frac{z^{n}}{n+\gamma+\beta}\right)
$$

is convex in $E$.

Since $f \in T_{\alpha, 2}\left[A, B ; 0 ; B_{1}\right]$, there exists $\psi_{1}=z \psi^{\prime} \in R_{\alpha, 2}[A, B]$

such that $\frac{f^{\prime}}{\psi^{\prime}} \in P_{\alpha}\left[0, B_{1}\right], \psi=V_{\alpha, 2}[A, B]$ in $E$. Let

$$
G_{1}(z)=\left[(\beta+1) z^{-\beta} \int_{0}^{z} t^{\beta-1} \psi_{1}^{\gamma}(t) d t\right]^{\frac{1}{\gamma}}, \quad G_{1}=z G^{\prime} .
$$

We first show that $G \in V_{\alpha, 2}[A, B]$.

From (3.7), it follows that

$$
\left\{z^{\beta} G_{1}^{\gamma}(z)\right\}^{\prime}=z^{\beta-1}\left(\psi_{1}^{\gamma}(z)\right)
$$

That is

$$
\left(G_{1}^{\gamma}(z)\right)\left[\beta+\gamma H_{1}(z)\right]=\psi_{1}^{\gamma}(z), \quad H_{1}(z)=\frac{z G_{1}^{\prime}(z)}{G_{1}(z)}
$$

Logarithmic differentiation of (3.9) and simple computations give us

$$
H_{1}(z)+\frac{z H_{1}^{\prime}(z)}{\gamma H_{1}(z)+\beta}=\frac{z \psi_{1}^{\prime}}{\psi_{1}(z)} \prec\left(\frac{1+A z}{1+B z}\right)^{\alpha} \prec\left(\frac{1+A z}{1+B z}\right) .
$$


Now, using Theorem 3.3 of [7, p: 109], It follows from (3.10) that $H_{1} \in P[A, B]$ and $G_{1}=z G^{\prime}$ belongs to $R_{1,2}[A, B]=S^{\star}[A, B]$. Therefore $G \in V_{1,2}[A, B]=C[A, B]$.

From (3.4), we have

$$
\begin{aligned}
\frac{z F_{1}^{\prime}(z) F_{1}^{\gamma-1}(z)}{G_{1}^{\gamma}(z)} & =\frac{\phi_{\gamma, \beta}(z) * z\left(\frac{\psi_{1}(z)}{z}\right)^{\gamma}\left(z f^{\prime}(z) \cdot \frac{f^{\gamma-1}(z)}{\psi_{1}^{\gamma}(z)}\right)}{\phi_{\gamma, \beta}(z) * z\left(\frac{\psi_{1}(z)}{z}\right)^{\gamma}} \\
& =\frac{\phi_{\gamma, \beta}(z) * z\left(\frac{\psi_{1}(z)}{z}\right)^{\gamma} h(z)}{\phi_{\gamma, \beta}(z) * z\left(\frac{\psi_{1}(z)}{z}\right)^{\gamma}}, \quad h \in P_{\alpha}\left(B_{1}\right) .
\end{aligned}
$$

Since $h(z)$ is analytic in $E, h(0)=1$, and $\phi_{\gamma, \beta}(z)$ is convex, $\psi_{1} \in S^{\star}$, we use a result due to Ruscheweyh and Sheil-Small [17] to conclude that $\left(\frac{z F_{1}^{\prime} F^{\gamma-1}}{G_{1}^{\gamma}}\right)(E) \subset \bar{C} o h(E)$, where $\bar{C} o h(E)$ denotes convex hull of $h(E)$. This implies $F_{1} \in T_{1,2}\left[A, B ; 0 ; B_{1}\right]$ in $E$. or $\gamma=1$ in (3.4), we obtain the well known Bernardi integral operator, see [7].

Theorem 3.3. Let $F=f * g, f \in T_{\alpha, m}[A, B ; 0 ; B], B \neq 0$. Then with $\rho=\left(\frac{1-A}{1-B}\right)^{\alpha}$ and $\gamma=\frac{A-B}{3 B}$,

$$
\left(\frac{1}{1+B r}\right)^{\alpha} \frac{(1-B r)^{\gamma(1-\rho)\left(\frac{m}{4}+\frac{1}{2}\right)}}{(1+B r)^{\gamma(1-\rho)\left(\frac{m}{4}-\frac{1}{2}\right)}} \leq\left|F^{\prime}(z)\right| \leq \frac{(1+B r)^{\gamma(1-\rho)\left(\frac{m}{4}+\frac{1}{2}\right)}}{(1-B r)^{\gamma(1-\rho)\left(\frac{m}{4}-\frac{1}{2}\right)}} \cdot\left(\frac{1}{1-B r}\right)^{\alpha}
$$

$$
\begin{aligned}
& \frac{2^{\gamma(1-\rho)}}{a|B|}\left[G_{12}(a, b ; c ;-1)-r_{1}^{-a} G_{12}\left(a, b ; c ;-r_{1}\right)\right] \\
& \leq|F(z)| \\
& \leq \frac{2^{\gamma(1-\rho)}}{a|B|} \cdot\left[G_{12}(a, b ; c ;-1)-r_{2}^{-a} G_{12}\left(a, b ; c ;-r_{2}\right)\right],
\end{aligned}
$$

where $r_{1}=-r_{2}^{-1}=\frac{1+B r}{1-B r}, m \leq\left[\frac{4(1-\alpha)}{\gamma(1-\rho)}+2\right]$ and $a$ is given in (3.16).

Proof. We can write for $F \in T_{\alpha, m}[A, B ; 0 ; B]$,

$$
F^{\prime}(z)=G^{\prime}(z) h(z), \quad h \in P_{\alpha}\left[B_{1}\right], \quad G=\psi * g \in V_{\alpha, m}[A, B] .
$$

Since $h \in P_{\alpha}[B]$, it easily follows that

$$
\left(\frac{1}{1+B r}\right)^{\alpha} \leq|h(z)| \leq\left(\frac{1}{1-B r}\right)^{\alpha}
$$

From Theorem 2.4 (ii) and (3.12), the proof of (i) is established.

We know proceed to prove (ii).

Let $d_{r}$ denote the radius of the largest schlicht disc centered at the origin contained in the image of $|z|<r$ under $F(z)$. Then there is a point $z_{0},\left|z_{0}\right|=r$, such that $\left|F\left(z_{0}\right)\right|=d_{r}$. The ray from 0 to $F\left(z_{0}\right)$ lies entirely 
in the image and the inverse image of this ray is a curve in $|z|<r$.

Using (3.11), we have

$$
\begin{aligned}
d_{r}=\left|F\left(z_{0}\right)\right| & =\int_{C}\left|F^{\prime}(z)\right||d z|, \quad r=\frac{A-B}{2 B} \\
& \geq \int_{0}^{|z|}\left[\frac{\left.(1-B s)^{\gamma\left\{(1-\rho)\left(\frac{m}{4}+\frac{1}{2}\right)\right\}}\right] d s}{\left.(1+B s)^{\left\{\gamma(1-\rho)\left(\frac{m}{4}-\frac{1}{2}\right)+\alpha\right\}}\right]}\right. \\
& =\int_{0}^{|z|}\left[\left(\frac{1-B s}{1+B s}\right)^{\left\{\gamma(1-\rho)\left(\frac{m}{4}-\frac{1}{2}\right)+\alpha\right\}} \cdot(1-B s)^{\gamma(1-\rho-\alpha)}\right] d s,
\end{aligned}
$$

Let $\frac{1+B s}{1-B s}=t$. Then $\frac{2 B}{(1-B s)^{2}}=d t$, and $1-B s=\frac{2}{1+t}$. This implies $d s=\frac{2}{B}\left(\frac{1}{1+t}\right)^{2} d t$. Therefore, from (3.13), we have

$$
\begin{aligned}
\left|F\left(z_{0}\right)\right| \geq & \int_{1}^{\frac{1+B r}{1-B r}} t^{-\left\{(1-\rho)\left(\frac{m}{4}-\frac{1}{2}\right)-\alpha\right\}} \cdot\left(\frac{2}{1+t}\right)^{1-\rho-\alpha} \cdot \frac{2}{B}\left(\frac{1}{1+t}\right)^{2} d t \\
= & \frac{-2^{(1-\rho)}}{|B|}\left[\int_{0}^{\frac{1+B r}{1-B r}} t^{\gamma(1-\rho)\left(\frac{m}{4}-\frac{1}{2}\right)-\alpha} \cdot(1+t)^{\gamma(1-\rho-\alpha)} d t\right. \\
& \left.-\int_{0}^{1} t^{r(1-\rho)\left(\frac{m}{4}-\frac{1}{2}\right)+\alpha} \cdot(1+t)^{r(1-\rho-\alpha)} d t\right] \\
= & \frac{2^{\gamma(1-\rho)}}{|B|}\left[I_{1}+I_{2}\right] .
\end{aligned}
$$

Now put $t=r_{1} u$ with $r_{1}=\frac{1+B r}{1-B r}$. Then $d t=r_{1} d u$ and

$$
\begin{aligned}
I_{1} & =\int_{0}^{1}\left(r_{1} u\right)^{-\left[\gamma(1-\rho)\left(\frac{m}{4}-\frac{1}{2}\right)-\alpha\right]} \cdot\left(1+r_{1} u\right)^{1-\rho-\alpha} d u \\
& =r_{1}^{-\left\{\gamma(1-\rho)\left(\frac{m}{4}-\frac{1}{2}\right)+\alpha-1\right\}} \int_{0}^{1} u^{-\gamma(1-\rho)\left(\frac{m}{4}-\frac{1}{2}\right)-\alpha} \cdot\left(1+r_{1} u\right)^{-\{\gamma(1-\rho)+\alpha\}} d u \\
& =r_{1}^{-\left\{\gamma(1-\rho)\left(\frac{m}{4}-\frac{1}{2}\right)+\alpha-1\right\}} \cdot \frac{\Gamma(a) \Gamma(c-a)}{\Gamma(c)} G_{12}\left(a, b ; c ;-r_{1}\right),
\end{aligned}
$$

where $\Gamma$ and $G_{12}$, respectively denote gamma and Gauss hypergeometric functions. Also, here, $b, c$ are positively real for $m \leq 2\left\{1+\frac{2(1-\alpha)}{1-\rho}\right\}$ and are given as

$$
\begin{aligned}
& a=-\gamma(1-\rho)\left(\frac{m}{4}-\frac{1}{2}\right)-\alpha+1, \quad \gamma=\frac{A-B}{2 B}, \quad B \neq 0 \\
& b=-\gamma(1-\rho)+\alpha, \\
& c=-\gamma(1-\rho)\left(\frac{m}{4}-\frac{1}{2}\right)-\alpha+2, \quad(c-a)>0 .
\end{aligned}
$$

Similarly, we calculate $I_{2}$ and have

$$
I_{2}=\frac{\Gamma(a) \Gamma(c-a)}{\Gamma(c)} G_{12}(a, b ; c ;-1) .
$$


Using (3.15), (3.16) and (3.17) in (3.14), we obtain the lower bound of $|F(z)|$. For the upper bound, we proceed in similar way and have

$$
\begin{aligned}
|F(z)| & \leq \int_{0}^{|z|} \frac{(1+B s)^{\gamma(1-\rho)\left(\frac{m}{4}+\frac{1}{2}\right)}}{(1-B s)^{\gamma(1-\rho)\left(\frac{m}{4}-\frac{1}{2}\right)}} \cdot\left(\frac{1}{1-B s}\right)^{\alpha} d s \\
& =\int_{0}^{|z|}\left(\frac{1+B s}{1-B s}\right)^{\gamma(1-\rho)\left(\frac{m}{4}-\frac{1}{2}\right)+\alpha} \cdot(1+B s)^{(1-\rho-\alpha)} d s .
\end{aligned}
$$

Now similar computations yield the required bound and the proof is complete.

By choosing suitable and permissible values of involved parameters, we obtain several new and also known results.

Remark 3.2. $\quad$ (i) We use a result of Pommerenke [16] together with

Theorem 3.1 and easily deduce that the class $T_{\alpha, m}[A, B ; 0 ;-1]$,

$m \leq 2\left\{1+\frac{\rho_{1}}{1-\rho}\right\}, \quad \rho_{1}=\left(\frac{1}{2}\right)^{\alpha}, \quad \rho=\left(\frac{1-A}{1-B}\right)^{\alpha}$, is a linearly invariant family of order $B_{2}=$ $\left\{\frac{m}{2}(1-\rho)+\left(\rho-\rho_{1}\right)+1\right\}$. With similar argument given in [16], we have the covering result for $T_{\alpha, m}[A, B ; 0 ;-1]$ as:

The image of $E$ under $F=f * g \in T_{\alpha, m}[A, B ; 0 ;-1]$ contains the Schlicht disc $|z|=\frac{1}{2 B_{2}}$, where $B_{2}=\left\{\frac{m}{2}(1-\rho)+1+\rho-\rho_{1}\right\}$, and $F(z)=z+\sum_{n=2}^{\infty} A_{n} z^{n}$.

(ii) Let $F_{*}$ be defined as

$$
F_{*}(z)=\frac{1}{B_{1}}\left[\left(\frac{1+z}{1-z}\right)^{B_{2}}-1\right]=z+\sum_{n=2}^{\infty} A_{n}^{*} z^{n}
$$

where

$$
\begin{aligned}
& B_{1}=\left\{\frac{m}{2}(1-\rho)+\left(\rho-\rho_{1}\right)+2\right\} \\
& B_{2}=\left\{\frac{m}{2}(1-\rho)+\left(\rho-\rho_{1}\right)+1\right\} .
\end{aligned}
$$

It can be shown, with some computations, that $F_{*}$ belongs to the linearly invariant family of $T_{\alpha, m}[A, B ; 0 ;-1]$.

Using this concept, together with the same argument of Pommerenke [16], we have $\left|A_{n}\right| \leq\left|A_{n}^{*}\right|, n \geq 1$ and $L_{r}(F) \leq L_{r}\left(F_{*}\right), F \in T_{\alpha, m}[A, B ; 0 ;-1]$ when $L_{r}(F)$ is the length of the image of the circle $|z|=r$ under $F, 0 \leq r<1$.

Theorem 3.4. Let $f \in T_{\alpha, m}[A, B ; 0 ;-1 ; g]$ and let $F=f * g \neq 0$ in $E$ with

$$
F(z)=z+\sum_{n=2}^{\infty} A_{n} z^{n}
$$

Then, for $m>2$,

$$
A_{n}=O(1) \cdot n^{\gamma_{1}}, \quad \gamma_{1}=\left\{\frac{m}{2}(1-\rho)+\left[\rho_{1}-(1+\rho)\right]\right\},
$$

where $O(1)$ is a constant depending on $m, \alpha, A$ and $B$ only. 
Proof. For $F \in T_{\alpha, m}[A, B ; 0 ;-1]$, we can write

$$
F^{\prime}=G^{\prime} h, \quad G \in V_{\alpha, m}[A, B], \quad G=\psi * g, \quad \psi \in V_{\alpha, m}[A, B ; g]
$$

Since $V_{\alpha, m}[A, B] \subset V_{m}(\rho), \rho=\left(\frac{1-A}{1-B}\right)^{\alpha}$, and it is well known that there exists $G_{i} \in V_{m}$ such that $G^{\prime}(z)=\left(G^{\prime}(z)\right)^{1-\rho}$ for $z \in E$.

Also $h \prec\left(\frac{1}{1+z}\right)^{\alpha}$, which implies $|\arg h(z)|<\frac{\rho_{1} \pi}{2}, \rho_{1}=\left(\frac{1}{2}\right)^{\alpha}$.

Therefore we have

$$
F^{\prime}=\left(G_{1}^{\prime}\right)^{1-\rho}\left(h_{1}\right)^{\rho_{1}}, \quad R e h_{1}>0
$$

in $E$.

For $G_{1} \in V_{m}$, there exists $s \in S^{\star}$ such that $G_{1}^{\prime}=s h_{2}^{\left(\frac{m}{2}-1\right)}, m>2$ and

$\operatorname{Reh}_{2}>0$ in $E$, see [1].

Thus, for $F \in T_{\alpha, m}[A, B ; 0 ;-1]$, it follows that

$$
F^{\prime}=(s)^{1-\rho}\left(h_{2}\right)^{(1-\rho)\left(\frac{m}{2}-1\right)}\left(h_{1}\right)^{\rho_{1}}, \quad h_{i} \in P, \quad i=1,2
$$

So, by Cauchy Theorem and (3.18), we have for $z=r e^{\iota \theta}$.

$$
\begin{aligned}
n\left|A_{n}\right| & \leq \frac{1}{2 \pi r^{n}} \int_{0}^{2 \pi}|s|^{1-\rho}\left|h_{1}\right|^{\rho_{1}}\left|h_{2}\right|^{(1-\rho)\left(\frac{m}{2}-1\right)} d \theta \\
& \leq \frac{1}{r^{n}}\left(\frac{r}{(1-r)^{2}}\right)^{(1-\rho)}\left[\left(\frac{1}{2 \pi} \int_{0}^{2 \pi}\left|h_{1}\right|^{2} d \theta\right)^{\frac{\rho_{1}}{2}} \cdot\left(\frac{1}{2 \pi} \int_{0}^{2 \pi}\left|h_{2}\right|^{\frac{2 \delta}{2-\rho_{1}}} d \theta\right)^{\frac{2-\rho_{1}}{2}}\right],
\end{aligned}
$$

where $\delta=(1-\rho)\left(\frac{m}{2}-1\right)$ and we have used distortion result for $s \in S^{\star}$ and Holder inequality.

Now, for $m>\left\{2+\frac{2-\rho_{1}}{1-\rho}\right\}$, we apply a result due to Hayman [5] for $h_{i} \in P$ and obtain

$$
n\left|A_{n}\right| \leq c\left(\rho, \rho_{1}, m\right) \cdot\left(\frac{1}{1-r}\right)^{1+\delta+\rho_{1}-2 \rho}
$$

where $c\left(\rho, \rho_{1}, \delta\right)$ is a constant.

Setting $r=1-\frac{1}{n}, n \rightarrow \infty$ in (3.19), the required result follows as

$$
A_{n}=O(1) \cdot n^{\left\{\frac{m}{2}(1-\rho)+\left[\rho_{1}-(\rho+1)\right]\right\}}, \quad \rho_{1}=\left(\frac{1}{2}\right)^{\alpha}, \quad \rho=\left(\frac{1-A}{1-B}\right)^{\alpha},
$$

and $m>\left\{2+\frac{2-\rho_{1}}{1-\rho}\right\}, \quad n \geq 2$.

Special Cases.

(i) $A=1$ implies that $\rho=0$ and for $\alpha=1, \rho_{1}=\frac{1}{2}$. Then

$$
A_{n}=O(1) \cdot n^{\frac{m}{2}-\frac{1}{2}}, \quad m>\frac{7}{2}
$$

Taking $m=4$, we have $A_{n}=O(1) \cdot n^{\frac{3}{2}}$. 
(ii) $A=\frac{1}{2}, B=-1, \alpha=1 \Rightarrow \rho=\frac{1}{4}$. Also $\rho_{1}=\frac{1}{2}$. Then $m=5>4$ implies $A_{n}=O(1) \cdot n^{\frac{9}{8}}$.

Acknowledgement: This research was supported by HEC Pakistan under project No: 8081/Punjab/NRPU /R\&D/HEC/2017.

Conflicts of Interest: The author(s) declare that there are no conflicts of interest regarding the publication of this paper.

\section{REFERENCES}

[1] D. A. Brannan, J. G. Clunie and W. E. Kirwan, On the coefficient problem for the functions of bounded boundary rotation, Ann. Acad. Sci. Fenn. Series AI Math. 523(1973), 1-18.

[2] D. A. Brannan, On functions of bounded boundary rotation, Proc. Edinburgh Math. Soc. 16(1969), 339-347.

[3] A. W. Goodman, Univalent Functions, Vol 1, 11, Polygonal Publishing House, Washington, New Jersey, 1983.

[4] A. W. Goodman, on Close-to-Convex Functions of Higher order, Ann. Univ. Sci. Budapest, Evotous Sect. Math. 25(1972), $17-30$.

[5] W. K. Hayman, on functions with positive real part, J. Lond. Math. Soc. 36(1961), 34-48.

[6] W. Janowski, Some extremal problems for certain families of analytic functions, Ann. Polon. Math. 28(1973), 297-326.

[7] S. S. Miller and P. T. Mocanu, Differential Subordination: Theory and Applications, Dekker, New York, 2000.

[8] K. I. Noor, On a generalization of Close-to-Convexity, Int. J. Math. Math. Sci. 6(1983), 327-334.

[9] K. I. Noor, Some properties of certain classes of functions with bounded radius rotation, Honam Math. J. 19(1997), 97-105.

[10] K. I. Noor, B. Malik and S. Mustafa, A survey on functions of bounded boundary and bounded radius rotation, Appl. Math. E-Notes, 12(2012), 136-152.

[11] K. I. Noor, On some univalent integral operations, J. Math. Anal. Appl. 128(1987), 586-592.

[12] K. I. Noor, Higher order close-to-convex functions, Math. Japonica, 37(1992), 1-8.

[13] K. Padmanabhan and R. Parvatham, Properties of a class of functions with bounded boundary rotation, Ann. Polon Math. 31(1975), 311-323.

[14] R. Parvatham and S. Radha, On certain classes of analytic functions, Ann. Polon Math. 49(1988), 31-34.

[15] B. Pinchuk, Functions with bounded boundary rotation, Isr. J. Math. 10(1971), 7-16.

[16] Ch. Pommerenke, Linear-invarient families analytischer funktionen 1, Math. Ann. 155(1964), 108-154.

[17] S. Ruscheweyh and T. Sheil-Small, Hadamard products of Schlicht functions and the Polya-Shoenberg conjecture, Comment. Math. Helv. 48(1973), 119-135. 